\title{
Klebsiella pneumoniae carbapenemase (KPC)-producing Klebsiella pneumoniae ST258 isolated from a Japanese patient without a history of foreign travel - a new public health concern in Japan: a case report
}

\author{
Yusuke Ainoda ${ }^{{ }^{*}}$ (D, Kotaro Aoki², Yoshikazu Ishii ${ }^{2}$, Kentaro Okuda $^{3}$, Hitomi Furukawa ${ }^{3}$, Ryo Manabe ${ }^{3}$, \\ Toshinori Sahara', Fukumi Nakamura-Uchiyama', Hitomi Kurosu', Yukiko Ando ${ }^{4}$, Maki Fujisawa ${ }^{4}$, Hitomi Hoshino ${ }^{4}$, \\ Hideki Arima ${ }^{4}$ and Kenji Ohnishi ${ }^{1}$
}

\begin{abstract}
Background: Thus far, studies on Klebsiella pneumoniae carbapenemase (KPC)-producing organisms have only been reported in those with a history of foreign travel, and a specific Japanese KPC-producing isolate has not yet been reported.

Case presentation: We describe a Japanese patient, with no history of travel to foreign countries, admitted due to aspiration pneumonia, and a KPC-producing isolate detected in his sputum. Fortunately, his pneumonia resolved. His close contacts did not have a history of foreign travel, and the isolate was not detected in other patients.

Conclusions: The potential for KPC-producing organisms to become endemic in Japan is currently of great concern.

Keywords: Klebsiella pneumoniae carbapenemase (KPC)-producing Klebsiella pneumoniae, K. pneumoniae ST258, Japanese
\end{abstract}

\section{Background}

Klebsiella pneumoniae carbapenemase (KPC)-producing Enterobacteriaceae have been reported in many countries, and outbreaks due to KPC-producing organisms have been reported worldwide [1-3]. KPC is an enzyme that degrades penicillin, cephalosporin, and broadspectrum $\beta$-lactams such as the carbapenems. This type of $\beta$-lactamase was discovered relatively recently, first detected in a strain isolated in the United States in 1996 [2]. The strain was considered a novel $\beta$-lactamase able to degrade carbapenem antibiotics; it was classified as a Bush group $2 \mathrm{f}$, class $A$, serine- $\beta$-lactamase and was

\footnotetext{
* Correspondence: surf_casting01@yahoo.co.jp

${ }^{1}$ Department of Infectious Diseases, Tokyo Metropolitan Health and Hospitals Corporation Ebara Hospital, 4-5-10 Higashi-Yukigaya Ota-ku, Tokyo 145-0065, Japan

Full list of author information is available at the end of the article
}

named KPC [4]. Because the mortality from infection by $\mathrm{KPC}$-producing organisms is reportedly higher than that of non-KPC-producers, these isolates have received a great deal of attention worldwide [5]. The most common carbapenemase gene to be reported in Japan is the IMP-type metallo- $\beta$-lactamase encoding gene [6] and the most common types are bla IMP-1 and $b l a_{\mathrm{IMP}-6}$. However, in Japan, KPC-producing organisms have so far only been reported as infections in patients who had visited foreign countries wherein KPC-producing organisms have been reported [7]. We report a Japanese patient without a history of travel to foreign countries whose sputum samples tested positive for a KPC producing isolate. The current report highlights a new concern regarding KPC-producing organisms that may already be present in Japan. 


\section{Case presentation}

A man in his early 90s was undergoing a follow-up for mild idiopathic interstitial pneumonia. He required assistance to perform activities of daily living, spent most of the day at home, and received periodic home visits for medical care. His last hospitalization was in February 2016 for approximately 1 month due to aspiration pneumonia. Only oral commensal bacteria were cultured from his sputum during his last hospitalization. In July 2016, he was hospitalized again for aspiration pneumonia. The sputum smears obtained on the first day of admission showed the presence of polymicrobial, normal oral bacteria and polymorphonuclear leukocytes. Subsequent cultures from this sputum showed normal oral bacteria as well as a few K. pneumoniae, with high levels of resistance to all antimicrobial agents except for minocycline. Results of examination of blood culture obtained on admission were negative. Other cultures were not examined. The patient had no history of travel to other countries and had never left Japan. Ampicillin/sulbactam was started at the time of hospitalization. On the 4th day of hospitalization, the antimicrobial agent was changed to cefepime because the clinical course was exacerbated. After the 5th day, the patient's clinical course improved, and this treatment was continued until the 12th day. The antimicrobial was not changed when $K$. pneumoniae was observed on a sputum culture collected on admission. The patient was discharged after his aspiration pneumonia had been successfully treated. Despite administering antibiotics that are generally not effective against K. pneumoniae, $K$. pneumoniae was not detected from his sputum after treatment.

\section{Laboratory investigation}

During laboratory investigation, we found that gramnegative bacillus grew on 5\% sheep blood agar. Carbapenem-resistant $K$. pneumoniae was identified by Phoenix100 and NMIC/ID-208 panel (Becton, Dickinson and Company). Minimum inhibitory concentration of both meropenem and imipenem was $>8 \mu \mathrm{g} / \mathrm{ml}$, and the sodium mercaptoacetate disk test result was negative. The modified Hodge test (using ertapenem disk) result was positive for $K$. pneumoniae TUM16641. The DNA of $K$. pneumoniae TUM16641 was sequenced using MiSeq (Illumina, Inc., CA, USA), and the DNA library for Illumia MiSeq sequencing was prepared using the Nextera XT Library Prep Kit (Illumina). The Nextera XT DNA library was sequenced in a paired-end $300 \mathrm{cy}-$ cles mode on MiSeq using 600 cycles Reagent Kit v3 (Illumina). Draft genomes (contigs) were obtained using CLC Genomics Workbench (Qiagen). TUM16641 belonged to sequence type (ST) 258 analyzed by multilocus sequence typing. A carbapenemase gene, $b l a_{\mathrm{KPC}-2}$, was detected in the contigs. To characterize a $b l a_{\mathrm{KPC}-2}$ carrying plasmid, we used a long reads sequencing platform, MinION (Oxford Nanopore Technologies [ONT], Oxford Science Park, UK). A MinION library was prepared from $K$. pneumoniae TUM16641 genomic DNA using Ligation Sequencing Kit 1D (SQK-LSK108) and Native Barcoding Kit (EXP-NBD103) (ONT). The

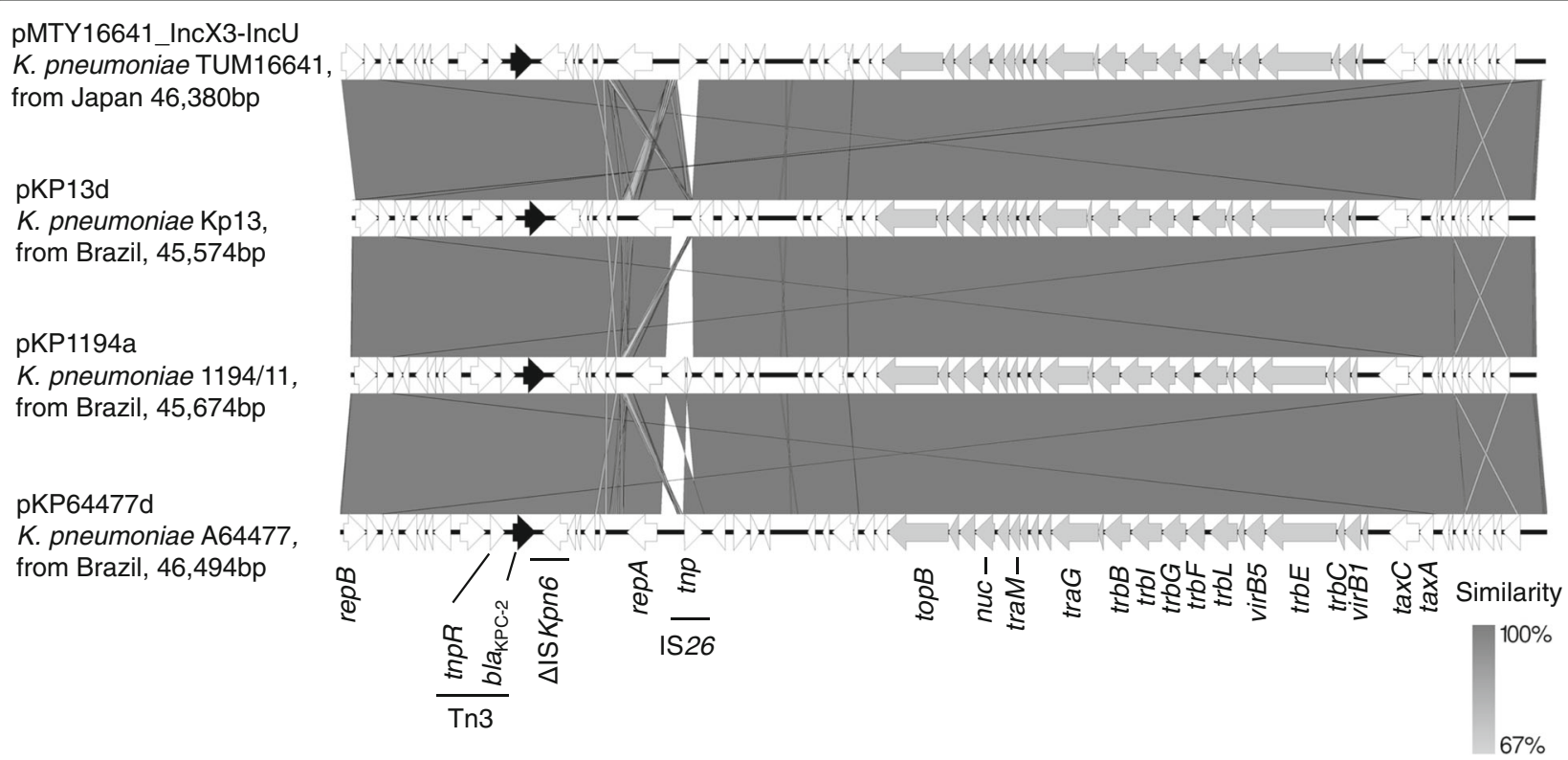

Fig. 1 Comparison of pMTY16641_IncX3_InCU (BFCA01000004) carrying blakPC-2 with pKP13d (CP003997), pKP1194a (KX756453), and pKP64477d (MF150120) drawn with EasyFig 2.1. All four plasmids were hybrid replicons of IncX3 and IncU. Arrow size is proportional to the predicted ORF length. The color code is as follows: The antibiotic resistance genes are in black and conjugal transfer genes are in gray. Others, putative, hypothetical, and unknown genes are represented by white arrows. Abbreviations: ORF: Open Reading Frame 
MinION DNA library was sequenced using Flow Cell R9.4 (FLOW-MIN106) (ONT). The complete plasmid sequence was obtained using SPAdes assemblers in combination with MiSeq and MinION data [8]. The sequencing data showed that the K. pneumoniae TUM16641 harbored a hybrid replicon of the IncX3 and IncU plasmid (pMTY16641_IncX3-IncU) carrying bla $a_{\mathrm{KPC}-2}$ (Fig. 1). The nucleotide sequence of pMTY16641_IncX3-IncU plasmid (GenBank accession number BFCA01000004) highly resembled that of pKP13d, pKP1194a, and pKP64477d of $K$. pneumoniae obtained from different reports in Brazil (Fig. 1). K. pneumoniae TUM16641 also harbored two antibiotic resistance gene carrying plasmids, a hybrid replicon of IncFIB and IncFII plasmid (pMTY16641_IncFIB-IncFII) carrying aadA2, aph(3')-Ia, mph(A), catA, sul1, and dfrA12 and a IncA/C2 plasmid (pMTY16641_ IncA/C2) carrying aac(3')-IId, rmtB, strA, strB, bla $a_{\text {TEM-1B }}$, bla $a_{\text {CTX-M-14, }}$ sul2, tet $(G)$ (Table 1).

The GenBank accession number for the draft wholegenome sequence data of the $K$. pneumoniae TUM16641 is DRR076334.

\section{Discussions and conclusions}

To our knowledge, this case showed the first possibility of a new threat in Japan regarding a KPC-producing isolate not linked with a history of travelling to other countries. The KPC-producing isolate was not detected in any other patients in the hospital where this patient was hospitalized previously; thus, the transmission route of this strain remains unclear. However, the present case suggests that KPC-producing organisms may be prevalent in Japan. Although no environmental culture was performed, the same bacteria were not observed in general culture tests of other patients who were hospitalized in the same medical institution. The origin of this isolate was unclear. This isolate was similar to a type reported in Brazil (assigned to the GenBank nucleotide sequence database under the accession number KU963389.1) and the USA [9]. The reported risks for infection by KPCproducing bacteria include patient immunodeficiency, use of intravascular devices, and use of various antimicrobial agents $[10,11]$. No intravascular devices and

Table 1 K. pneumoniae harboring replicons (chromosome and plasmid) and antibiotic resistance genes

\begin{tabular}{|c|c|}
\hline Replicon and plasmid Inc/Rep type & Antibiotic resistance genes \\
\hline Chromosome & bla $_{\mathrm{SHV}-11}$, oqxA, oqxB, fos $A$ \\
\hline IncFIB and IncFIl plasmid & $\begin{array}{l}\text { aadA2, aph(3')-la, mph(A), catA, sul1, } \\
\text { dfrA12 }\end{array}$ \\
\hline IncA/C2 plasmid & $\begin{array}{l}\text { aac( }\left(3^{\prime}\right)-11 d, r m t B, \operatorname{str} A, \operatorname{str} B, b l a \text { TEM-1B } \\
\text { bla }{ }^{a} \text { CTX-M-14, } \operatorname{sul} 2, \operatorname{tet}(G)\end{array}$ \\
\hline IncX3 and IncU plasmid & $b / a_{\mathrm{KPC}-2}$ \\
\hline ColRNAl plasmid & ND \\
\hline
\end{tabular}

${ }^{\mathrm{a}}$ Two $b / a_{\mathrm{TEM}-1 \mathrm{~B}}$ were located on IncA/C2 plasmid. ND: not detected no agents that could lead to immunodeficiency were used in this patient. However, considering that he was elderly, had experienced aspiration pneumonia, and had undergone 10 days of antibiotic treatment 5 months previously, the KPC-producing organism identified in his sputum may have been selected by exposure to ampicillin/sulbactam and meropenem. This patient's symptoms were relieved by treatment of his bacterial pneumonia. The patient was likely a carrier of a KPC-producing isolate, as he did not develop a K. pneumoniae infection. Clinical improvement without susceptible antimicrobial agents suggests the possibility that $K$. pneumoniae was not the causative organism in the present case.

We report a new public health concern in Japan regarding a KPC-producing isolate detected in a Japanese patient with no history of foreign travel. These findings highlight a growing concern regarding the potential spread of KPC-producing organisms, and that the possibility of detecting such bacteria should be considered even in Japan.

Abbreviation

KPC: Klebsiella pneumoniae carbapenemase

Acknowledgements

Not applicable.

Funding

There is no funding to declare in this study.

Availability of data and materials

The data that support the findings of this study are available from the corresponding author (YA) upon request.

\section{Authors' contributions}

YA1 (corresponding author) was in charge of infection control and drafted the manuscript. KA and $\mathrm{Yl}$ performed the whole-genome sequencing analysis of $K$. pneumoniae TUM16641 and helped with the draft of the manuscript. $\mathrm{KO}, \mathrm{HF}$ and RM treated the patient. TS, FNU and HK also coordinated infection control. YA2, MF, HH and HA performed biochemical tests for this strain. KO revised the article for intellectual content. All authors have read and critically revised the first as well as the subsequent and final drafts of this manuscript. All authors read and approved the final manuscript.

Ethics approval and consent to participate

The study was in accordance with the tenets of the Declaration of Helsinki. The requirement for approval from the Ethics Committee is unnecessary, based on the guidelines of the Ministry of Health, Labor and Welfare in Japan. The Ethics Committee at Tokyo Metropolitan Health and Medical Treatment Corporation Ebara Hospital ruled that no formal ethical approval was required for reporting this case.

Consent for publication

Written consent to publish the case report was obtained from the patient.

Competing interests

The authors declare that they have no competing interests.

\section{Publisher's Note}

Springer Nature remains neutral with regard to jurisdictional claims in published maps and institutional affiliations. 


\section{Author details}

'Department of Infectious Diseases, Tokyo Metropolitan Health and Hospitals Corporation Ebara Hospital, 4-5-10 Higashi-Yukigaya Ota-ku, Tokyo 145-0065, Japan. ${ }^{2}$ Department of Microbiology and Infectious Diseases, Toho University School of Medicine, 5-21-16 Omori-Nishi Ota-ku, Tokyo 143-8540, Japan. ${ }^{3}$ Department of Pulmonary Medicine, Tokyo Metropolitan Health and Hospitals Corporation Ebara Hospital, 4-5-10 Higashi-Yukigaya Ota-ku, Tokyo 145-0065, Japan. ${ }^{4}$ Department of Laboratory, Tokyo Metropolitan Health and Hospitals Corporation Ebara Hospital, 4-5-10 Higashi-Yukigaya Ota-ku, Tokyo 145-0065, Japan

Received: 3 April 2018 Accepted: 21 December 2018

\section{Published online: 07 January 2019}

\section{References}

1. Naas T, Nordmann P, Vedel G, Poyart C. Plasmid-mediated carbapenemhydrolyzing $\beta$-lactamase KPC in a Klebsiella pneumoniae isolate from France. Antimicrob Agents Chemother. 2005;49(10):4423-4.

2. Woodford N, Tierno PM Jr, Young K, Tysall L, Palepou MF, Ward E, et al. Outbreak of Klebsiella pneumoniae producing a new carbapenem-hydrolyzing class a beta-lactamase, KPC-3, in a New York medical center. Antimicrob Agents Chemother. 2004;48(12):4793-9.

3. Wei ZQ, Du XX, Yu YS, Shen P, Chen YG, Li LJ. Plasmid-mediated KPC-2 in a Klebsiella pneumoniae isolate from China. Antimicrob Agents Chemother. 2007;51(2):763-5.

4. Yigit $\mathrm{H}$, Queenan $\mathrm{AM}$, Anderson GJ, Domenech-Sanchez A, Biddle JW, Steward CD, et al. Novel carbapenem-hydrolyzing $\beta$-lactamase, KPC-1, from a carbapenem-resistant strain of Klebsiella pneumoniae. Antimicrob Agents Chemother. 2001;45(4):1151-61.

5. Marchaim D, Navon-Venezia S, Schwaber MJ, Carmeli Y. Isolation of imipenemresistant Enterobacter species: emergence of KPC-2 carbapenemase, molecular characterization, epidemiology, and outcomes. Antimicrob Agents Chemother. 2008:52(4):1413-8.

6. Yano H, Ogawa M, Endo S, Kakuta R, Kanamori H, Inomata S, et al. High frequency of IMP-6 among clinical isolates of metallo- $\beta$-lactamaseproducing Escherichia coli in Japan. Antimicrob Agents Chemother. 2012:56(8):4554-5.

7. Saito R, Takahashi R, Sawabe E, Koyano S, Takahashi Y, Shima M, et al. First report of KPC-2 carbapenemase-producing Klebsiella pneumoniae in Japan. Antimicrob Agents Chemother. 2014;58(5):2961-3.

8. Bankevich A, Nurk S, Antipov D, Gurevich AA, Dvorkin M, Kulikov AS, et al. J Comput Biol. 2012;19(5):455-77.

9. Ho PL, Cheung YY, Lo WU, Li Z, Chow KH, Lin CH, et al. Molecular characterization of an atypical IncX3 plasmid PKPC-NY79 carrying bla $a_{\mathrm{KPC}-2}$ in a Klebsiella pneumoniae. Curr Microbiol. 2013;67(4):493-8.

10. Nordmann P, Cuzon G, Naas T. The real threat of Klebsiella pneumoniae carbapenemase-producing bacteria. Lancet Infect Dis. 2009;9(4):228-36.

11. Woodford N, Tierno PM Jr, Young K, Tysall L, Palepou MF, Ward E, et al. Outbreak of Klebsiella pneumoniae producing a new carbapenem-hydrolyzing class a $\beta$-lactamase, KPC-3, in a New York medical center. Antimicrob Agents Chemother. 2004;48(12):4793-9.

\section{Ready to submit your research? Choose BMC and benefit from:}

- fast, convenient online submission

- thorough peer review by experienced researchers in your field

- rapid publication on acceptance

- support for research data, including large and complex data types

- gold Open Access which fosters wider collaboration and increased citations

- maximum visibility for your research: over $100 \mathrm{M}$ website views per year

At BMC, research is always in progress.

Learn more biomedcentral.com/submissions 